\title{
DIQUES MÁFICOS DE MINAS GERAIS DO CRETÁCEO INFERIOR: IDADES Ar-Ar E CORRELAÇÃO COM A PROVÍNCIA ÍGNEA PARANÁ- ETENDEKA
}

\author{
LOWER CRETACEOUS MAFIC DYKE SWARMS FROM MINAS GERAIS: Ar-Ar AGES AND \\ CORRELATION WITH PARANÁ-ETENDEKA IGNEOUS PROVINCE
}

\author{
Raphael Martins COELHO, Alexandre de Oliveira CHAVES \\ Universidade Federal de Minas Gerais. Emails: raphaelmcoelho@yahoo.com.br; alex2010@ufmg.br \\ Introdução \\ Geologia, aspectos magnetométricos e petrografia dos diques máficos mesozoicos de Minas Gerais \\ Metodologia \\ Resultados \\ Discussões e conclusões \\ Agradecimentos
}

\begin{abstract}
RESUMO - Durante o Cretáceo Inferior, houve um intenso evento magmático relacionado à fragmentação da parte oeste do supercontinente Gondwana, dando início ao desenvolvimento da bacia de margens continentais brasileira e africana e a abertura do Oceano Atlântico Sul. Este magmatismo é representado pelos derrames basálticos e enxames de diques máficos associados à enorme província ígnea Paraná (Brasil) - Etendeka (África) de 133 a 135 Ma. Destacando-se em imagens magnetométricas, no estado de Minas Gerais ocorrem vários enxames de diques com idades desde o Pré-Cambriano até o Cretáceo Inferior. Atravessando o Cráton do São Francisco e seus cinturões brasilianos, os diques Transminas e parte dos diques máficos Serra do Mar compõem os mais jovens enxames de Minas Gerais. Em Minas Gerais, idade plateau de 124.56 \pm 2.15 Ma e idade isocrônica inversa de $131.92 \pm 3.63$ Ma para um dique do enxame Serra do Mar, bem como idade plateau de $135.72 \pm 4.35$ Ma e idade isocrônica inversa de $128.43 \pm$ 8.46 Ma para um dique do enxame Transminas, obtidas no presente trabalho pelo método Ar-Ar, inserem ambos os enxames de Minas Gerais no mesmo contexto geotectônico de intrusão dos diques máficos Serra do Mar (fora de Minas Gerais), Ponta Grossa (Paraná), Florianópolis (Santa Catarina) e Vitória-Colatina (Espirito Santo), todos eles associados à Província Ígnea Paraná-Etendeka.

Palavras-chave: enxames de diques Serra do Mar e Transminas; Cretáceo; Província Ígnea Paraná-Etendeka.
\end{abstract}

\begin{abstract}
An intense Lower Cretaceous magmatic event have been related to break-up of the western Gondwana supercontinent, initiating the development of basin with Brazilian and African continental margins and the opening of the South Atlantic Ocean. This magmatism is represented by flood basalts and mafic dykes swarms associated with 133-135 Ma Parana (Brazil) - Etendeka (Africa) large igneous province. Revealed by magnetometric images and with ages from Precambrian to the Lower Cretaceous, several dyke swarms occur in the State of Minas Gerais (MG). Crossing the São Fransisco Craton and its surrounding Brasiliano Mobile Belts, the Transminas mafic dykes and a group of Serra do Mar mafic dykes represent the younger swarms of Minas Gerais. In Minas Gerais, plateau age of 124.56 \pm 2.15 Ma and inverse isochron age of $131.92 \pm 3.63$ Ma for a Serra do Mar dyke and plateau age of $135.72 \pm 4.35 \mathrm{Ma}$ and inverse isochron age of $128.43 \pm 8.46 \mathrm{Ma}$ for a Transminas dike, obtained in this research by the Ar-Ar method, insert both Cretaceous mafic dyke swarms in the same intrusion tectonic context of Serra do Mar (outside Minas Gerais), Ponta Grossa (Paraná), Florianópolis (Santa Catarina) and Vitória-Colatina (Espirito Santo) mafic dyke swarms, all of them associated with Paraná-Etendeka igneous province.
\end{abstract}

Keywords: Serra do Mar and Transminas mafic dyke swarms; Cretaceous; Paraná-Etendeka Igneous Province.

\section{INTRODUÇÃO}

Províncias Ígneas Gigantes (Large Igneous Provinces-LIP) compreendem massivos derrames de magmas basálticos e intrusões associadas de enxames de diques produzidos num intervalo relativamente curto de tempo ( $<10$ milhões de anos) e que se estendem por milhares de $\mathrm{km}^{2}$. Elas podem estar relacionadas à atividade de plumas mantélicas e grandes eventos de quebra e dispersão de massas continentais. Durante o Cretáceo, houve um intenso evento magmático relacionado à fragmentação da parte oeste do supercontinente Gondwana, dando início ao desenvolvimento das bacias de margens continentais brasileira e africana e a abertura do Oceano Atlântico Sul (Figuras 1A e 1B).
Este magmatismo é representado pela LIP Paraná-Etendeka, de idade U-Pb entre 135 e 133 Ma (Pinto et al., 2010), e pelos enxames de diques máficos a ela associados. Estes enxames afloram no Paraná (diques Ponta Grossa de 134 Ma - idade média de dados K-Ar e Ar-Ar calculada por Tomba (2012), nos estados de São Paulo e Rio de Janeiro (diques Serra do Mar ou Santos-Rio de Janeiro de 134 Ma idade média de dados K-Ar e Ar-Ar calculada por Tomba (2012), em Santa Catarina (diques Florianópolis de $134 \mathrm{Ma}, \mathrm{U}-\mathrm{Pb}$, (Florisbal et al., 2011) e no Espírito Santo (diques VitoriaColatina de 128-136 Ma, Ar-Ar, (Novais et al., 2003; Belém, 2014).

Muitos autores entendem que o mecanismo gerador da LIP Paraná-Etendeka está relacionado 
com a atividade da pluma mantélica Tristão da Cunha (Figura 1B) quando o supercontinente Gondwana derivou por sobre ela (White \&
McKenzie, 1989, Renne et al., 1992, O’Connor et al., 1990, Harry et al., 1992, Valente et al., 2007, 2009, Corval, 2009 e Chaves, 2014).

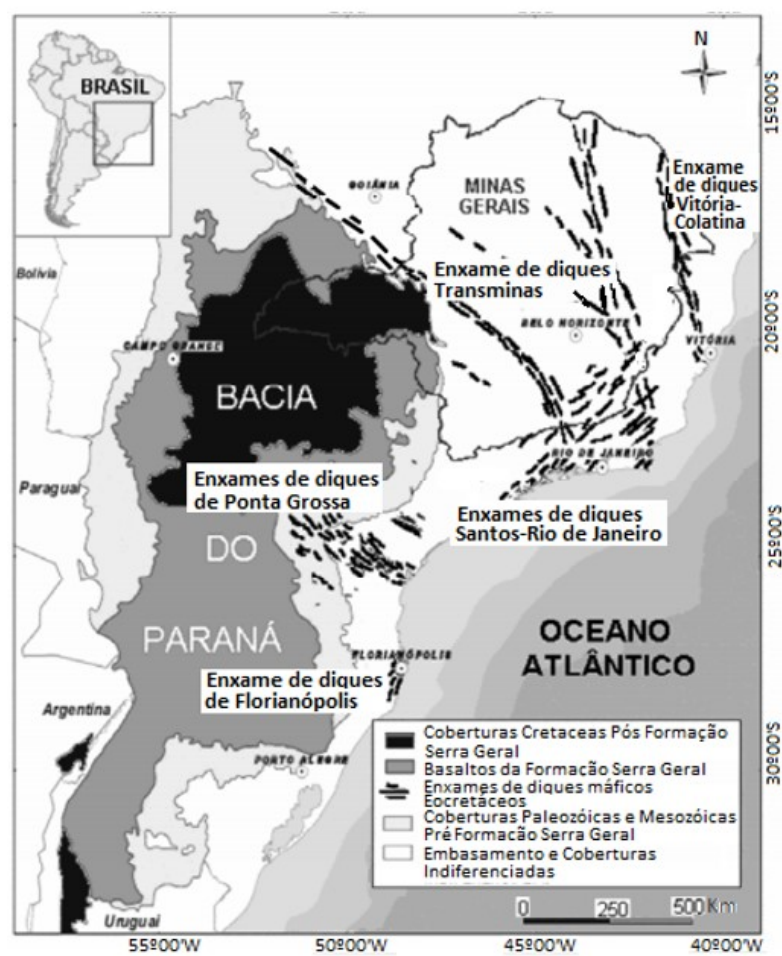

A
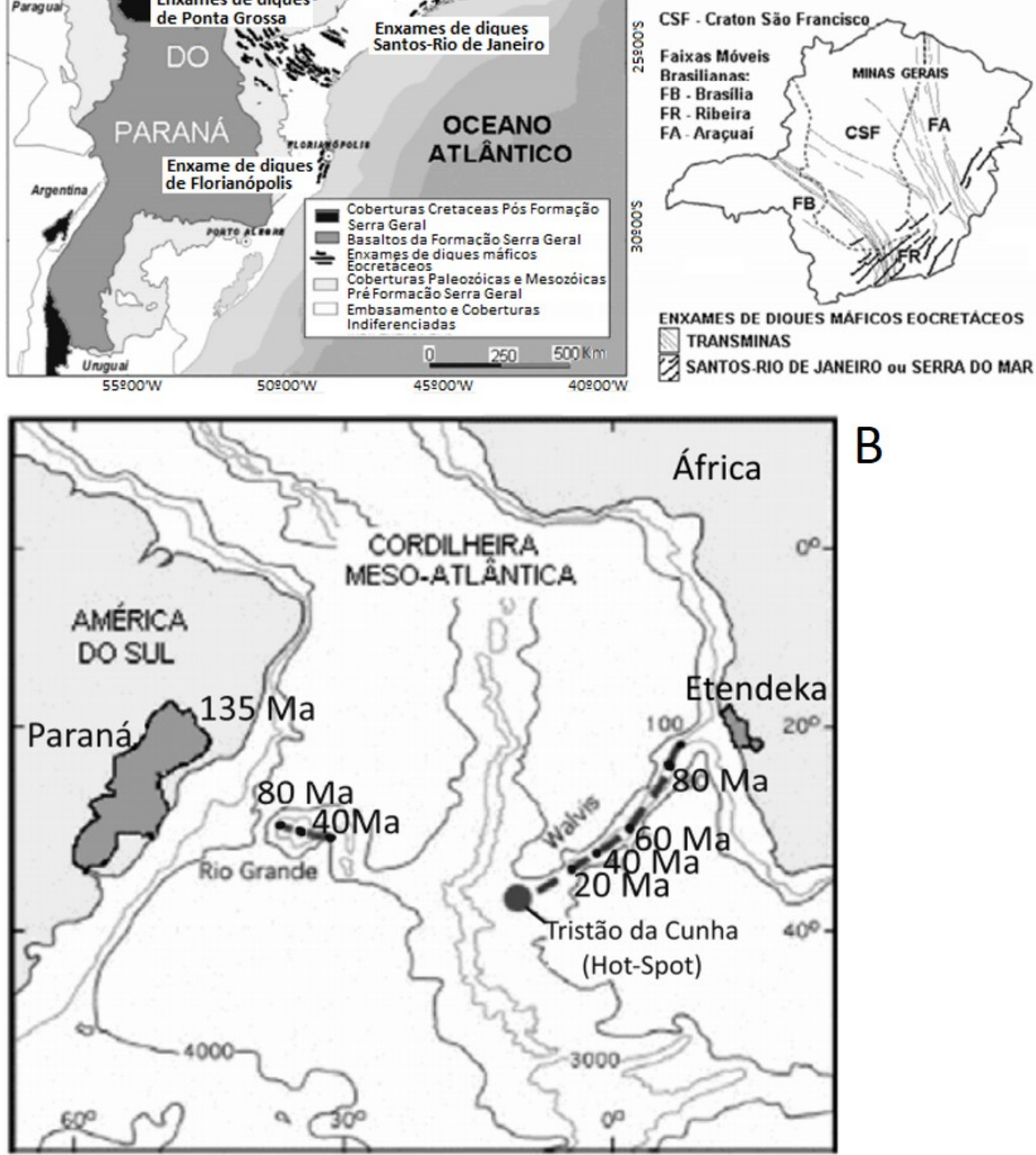

Figura 1 - A) Panorama geral da LIP Paraná-Etendeka e seus diques máficos associados, modificado de Tomba (2012) e Chaves (2014). B) Configuração atual da LIP Paraná-Etendeka e posição do Hot-Spot Tristão da Cunha com as idades nos altos do Rio Grande e Walvis, indicando idades decrescentes em direção ao Hot-Spot (White \& McKenzie, 1989, modificado).

Tendo em vista a dispersão nos valores de idades publicados na literatura para os diques máficos mesozoicos de Minas Gerais (170-220 Ma, K-Ar, Dussin et al., 1995, 120 Ma, K-Ar, Silva et al., 1995, 127 Ma, K-Ar, Pinese, 1997, $130 \mathrm{Ma}$, Ar-Ar, Rosset et al., 2007), o objetivo deste trabalho é apresentar novos dados geocronológicos pelo método $\mathrm{Ar}$-Ar para estes diques que permitam precisar o intervalo de idade de seu posicionamento crustal e discutir relações geodinâmicas destes diques com o magmatismo da LIP Paraná-Etendeka. 


\section{GEOLOGIA, ASPECTOS MAGNETOMÉTRICOS E PETROGRAFIA DOS DIQUES MÁFICOS MESOZOICOS DE MINAS GERAIS}

No estudo de enxames de diques máficos, uma ferramenta bastante eficaz são imagens magnetométricas, devido ao contraste magnético que estas rochas apresentam em relação às suas encaixantes. Destacando-se em imagens deste tipo, no estado de Minas Gerais ocorrem vários enxames de diques com idades desde o Pré Cambriano até o Cretáceo, ora atravessando o Cráton do São Francisco, ora atravessando seus cinturões brasilianos ou ambos.
Na figura 2, uma imagem magnetométrica obtida a partir de levantamentos aerogeofísicos (metodologia de obtenção de dados magnetométricos vide CPRM/CODEMIG, 2014) realizados pela Companhia de Desenvolvimento Econômico de Minas Gerais (CODEMIG), Serviço Geológico do Brasil (CPRM) e Agência Nacional do Petróleo (ANP), os lineamentos destacados em tons de vermelho e azul correspondem aos diques máficos mesozoicos.

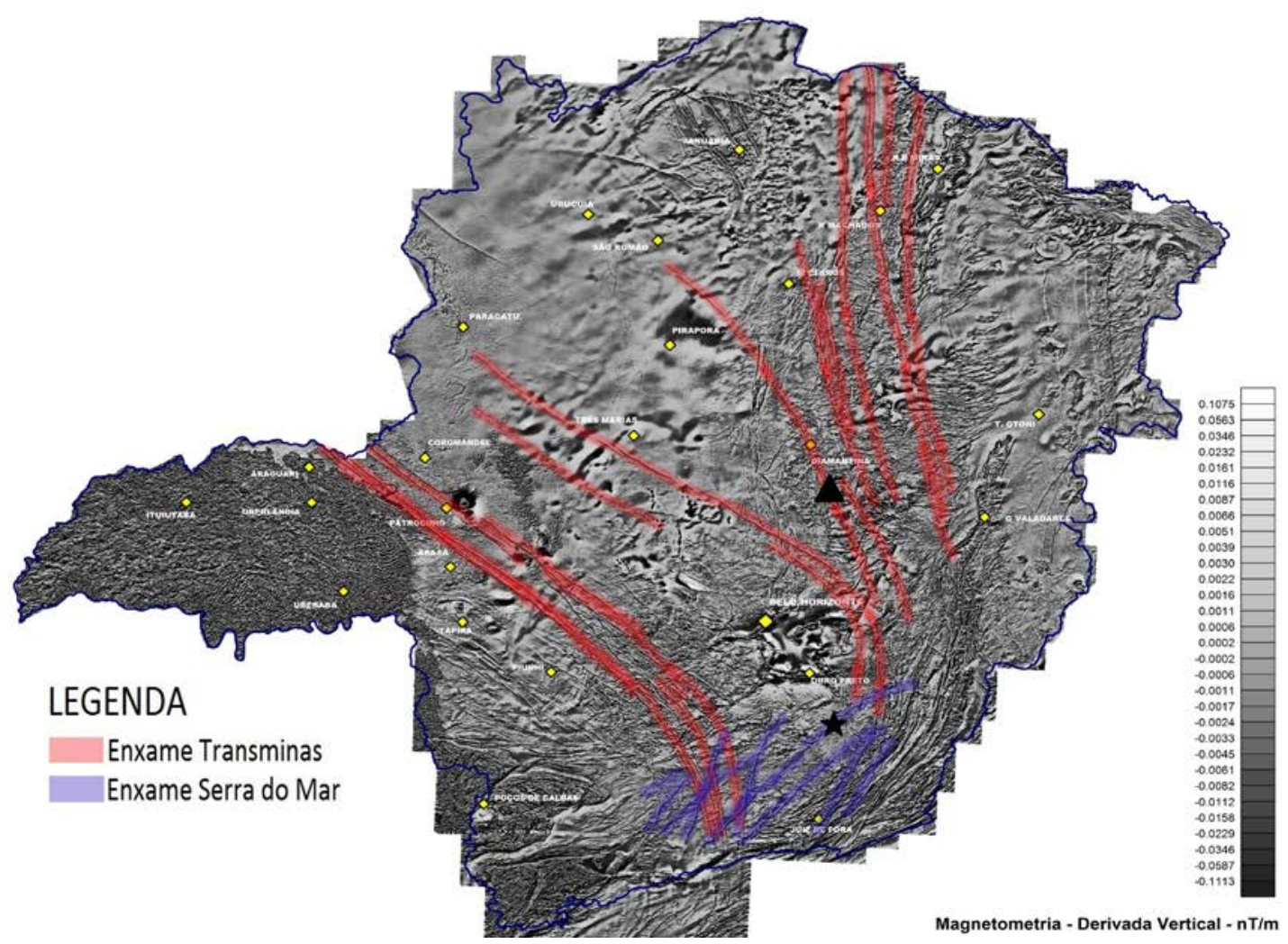

Figura 2 - Mapa magnetométrico do estado de Minas Gerais, destacando os lineamentos que correspondem aos diques máficos mesozoicos (CPRM/CODEMIG, 2014 modificado). O ponto de coleta da amostra 10A está representado pela estrela e da amostra 14, pelo triangulo.

Quando aflorantes, estas rochas normalmente apresentam-se superficialmente na forma de blocos centimétricos a métricos arredondados e alterados in situ ou bem preservadas em drenagens naturais e cortes de estradas. Pertencentes ao período mesozoico, em Minas Gerais afloram parte dos diques do enxame Serra do Mar e os diques do enxame Transminas (Chaves, 2013).

O enxame de diques Serra do Mar intrudem rochas do Orógeno Neoproterozoico/CambroOrdoviciano Ribeira (Heilbron et al., 2004; Schmitt et al., 2004) e rochas do
Arqueano/Paleoproterozoico do extremo sul do Cráton do São Francisco (Figuras 1 e 2). Valente (1997), Corval (2005) e Dutra (2006) mostraram que as rochas que constituem este enxame são compostas, essencialmente, por plagioclásio, clinopiroxênio e olivina. Minerais opacos, apatita e quartzo ocorrem como minerais acessórios.

Assim como nos estados de São Paulo e Rio de Janeiro, em Minas Gerais estas rochas são também compostas por plagioclásio e clinopiroxênio e cristais de olivina, em sua maioria substituída por iddingsita. Os opacos e a apatita são os minerais acessórios (Figura 3B). 
São diabásios de cor cinza esverdeada, de textura subofítica e granulação muito fina, holocristalinas com cristais hipidiomórficos. Ocorrem eventualmente amígdalas preenchidas por clorita e quartzo e nota-se sutil processo de argilização das ripas de plagioclásio. $\mathrm{O}$ enxame denominado Transminas (Chaves \& Neves, 2005, Chaves, 2013) atravessa todo o estado de Minas Gerais com direção NW-SE e mais de 500 km de extensão (Figuras 1 e 2). De acordo com o trabalho de Rocha (2013), dados magnetométricos mostram que o braço oeste deste

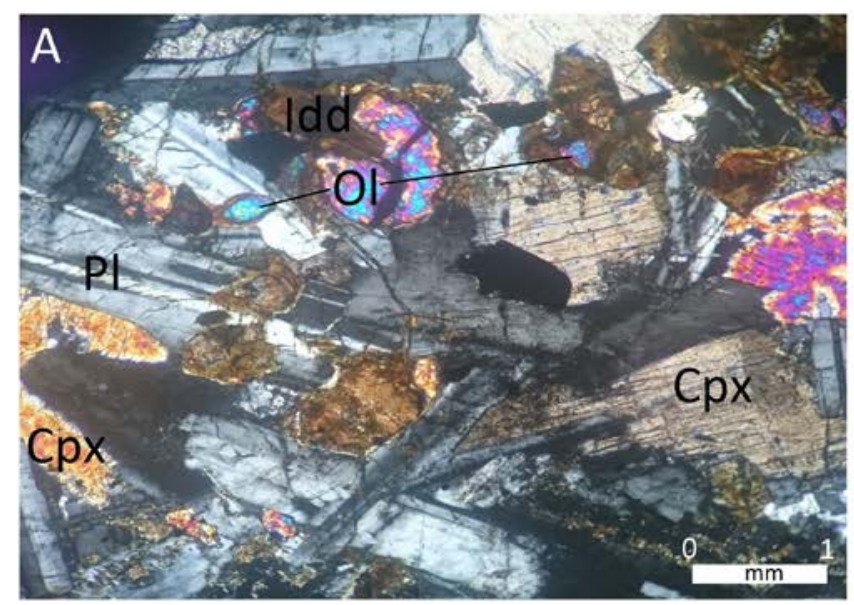

enxame de diques também corta parte do estado de Goiás, perfazendo um total de aproximadamente $850 \mathrm{~km}$.

Os diques do enxame Transminas atravessam não só o Cráton do São Francisco e suas coberturas pré-neocretáceas, como também as faixas móveis brasilianas Brasília, Ribeira e Araçuaí (Figura 1A). Suas rochas são diabásios com textura ofítica a subofítica. Quanto aos constituintes minerais, possuem plagioclásio, clinopiroxênio, olivina (em substituição para iddingsita), ilmenomagnetita e apatita (Figura 3A).

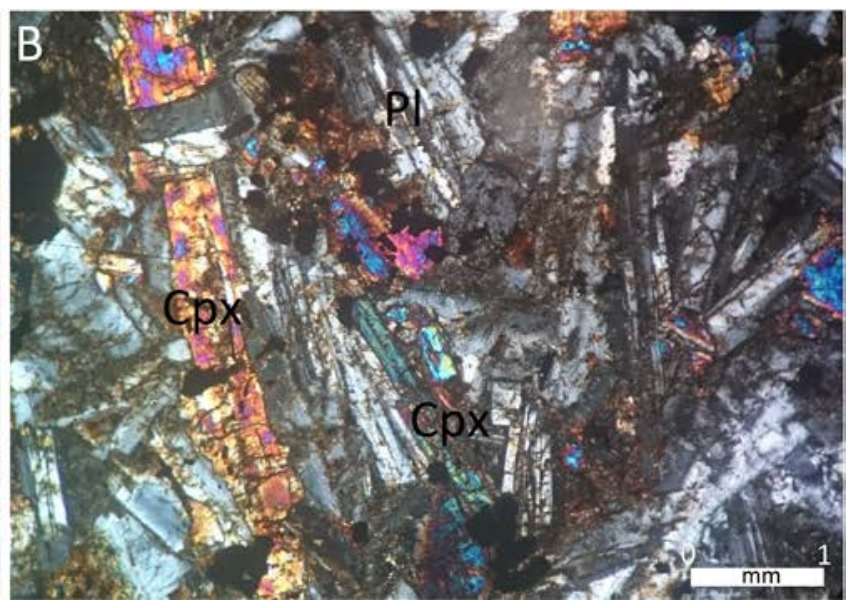

Figura 3 -Fotomicrografias das rochas estudadas, sob polarizadores cruzados. Em A, aparece a amostra 10A dos diques Serra do Mar e, em B, a amostra 14 dos diques Transminas. Cpx = Clinopiroxênio, $\mathrm{Pl}=$ Plagioclásio, $\mathrm{Ol}=$ Olivina In = Iddingsita. Em preto são os opacos.

\section{METODOLOGIA}

Duas amostras coletadas foram selecionadas para estudos geocronológicos, sendo uma do enxame Transminas (Coordenadas Lat.: -19.45684; Long.: 43.1096317, vide triângulo na figura 2) e a outra do enxame Serra do Mar (Coordenadas Lat.: -20.945873; Long.: -43.3731452, vide estrela na figura 2). Após separação granulométrica, fragmentos de rocha sem sinais de alteração intempérica foram selecionados para as análises isotópicas Ar-Ar.

A separação de minerais como o plagioclásio para datação não foi possível, pois a granulação muito fina das amostras de diabásio não permitiu que o mesmo fosse isolado de outros minerais como o piroxênio e a olivina.

Os fragmentos de rocha foram irradiados por fluxo de nêutrons epitermais rápidos no reator de pesquisa TRIGA (CLICIT) do OSU/Oregon (USA) durante 15 horas contínuas, juntamente com o padrão internacional (monitor de fluxo) Fish Canyon (sanidina, $28.305 \pm 0.036 \mathrm{Ma}$;
Renne et al. 2010).

Após um período de aproximadamente 20 dias de resfriamento e cerca de 30 dias de deslocamento USA/Brasil, a amostra foi depositada para análise por aquecimento gradual (step heating) via laser de estado sólido Nd:YVO4 (visível próximo, $532 \mathrm{~nm}$ - modelo Verdi 6W Coherent) no Laboratório de $\mathrm{Ar}$ do Centro de Pesquisas Geocronológicas da Universidade de São Paulo (CPGeo/IGc/USP). O sistema de extração a laser se encontra acoplado a um sistema de purificação funcionando com getters SAES-GP50 e espectrômetro de massa multicoletor ARGUS VI (ThermoScientific, Bremen, Alemanha), de setor magnético.

As massas 40 a 36 foram medidas simultaneamente em 5 coletores Faraday (1011 ohm - para a massa 40, e 1012 ohm para as demais massas). A reprodutibilidade durante a sequência de análises (run SP2) foi monitorada continuamente através de medidas de $\mathrm{Ar}$ atmosférico (air pippete, ${ }^{40} \mathrm{Ar} /{ }^{36} \mathrm{Ar}$ 
weighted mean $=298.5)$. As idades aparentes foram calculadas a cada etapa de aquecimento usando um fator $\mathrm{J}=0.00419 \pm 0.00002$ ), após serem aplicadas as correções para discriminação de massa, interferências nucleogênicas e contaminação atmosférica.

\section{RESULTADOS}

A fração de rocha total da amostra 10A (diques Serra do Mar) foi analisada durante 19 etapas de aquecimento, com intervalo de $0.1 \mathrm{~W}$ para a potência do laser aplicada. As frações de gás liberadas produziram idades aparentes muito coerentes entre si, a $2 \sigma$ de incerteza. Como consequência, o gráfico de aquecimento gradual mostra um plateau constante com idade de $124.56 \pm 2.15$ Ma (erro de $1.73 \%, 2 \sigma$, com $53.7 \% \mathrm{de}^{39} \mathrm{Ar}$ liberado) conforme a figura $4 \mathrm{~A}$.

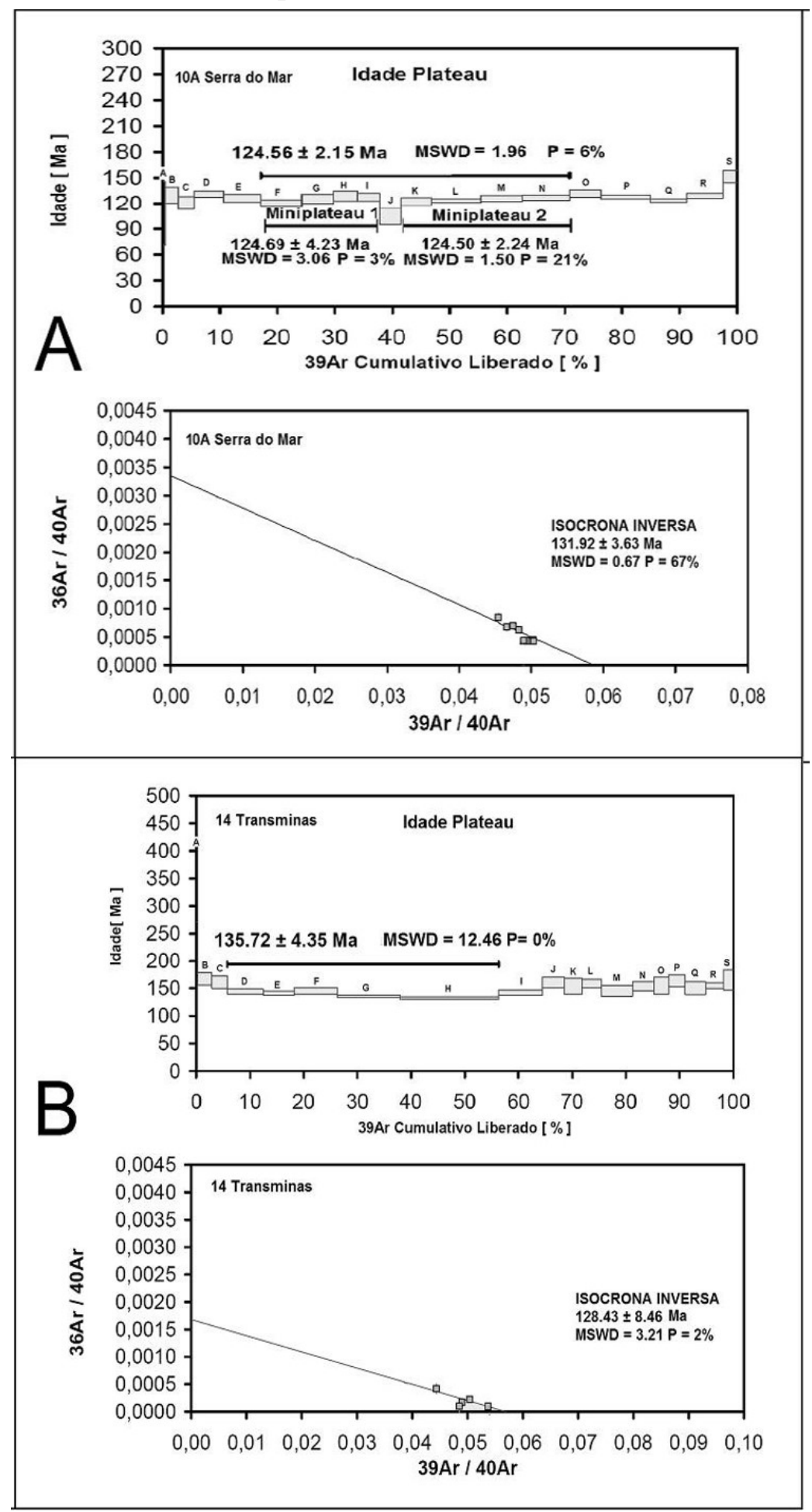

Figura 4 - Gráficos de idades Ar-Ar para os diques mesozoicos de Minas Gerais. A) Gráficos do dique Serra do Mar (amostra10A). B) Gráficos do dique Transminas (amostra 14). 
Tabela 1 - Resultados das análises isotópicas Ar-Ar para a amostra 10A e 14 (em negrito, os dados utilizados na construção dos gráficos).

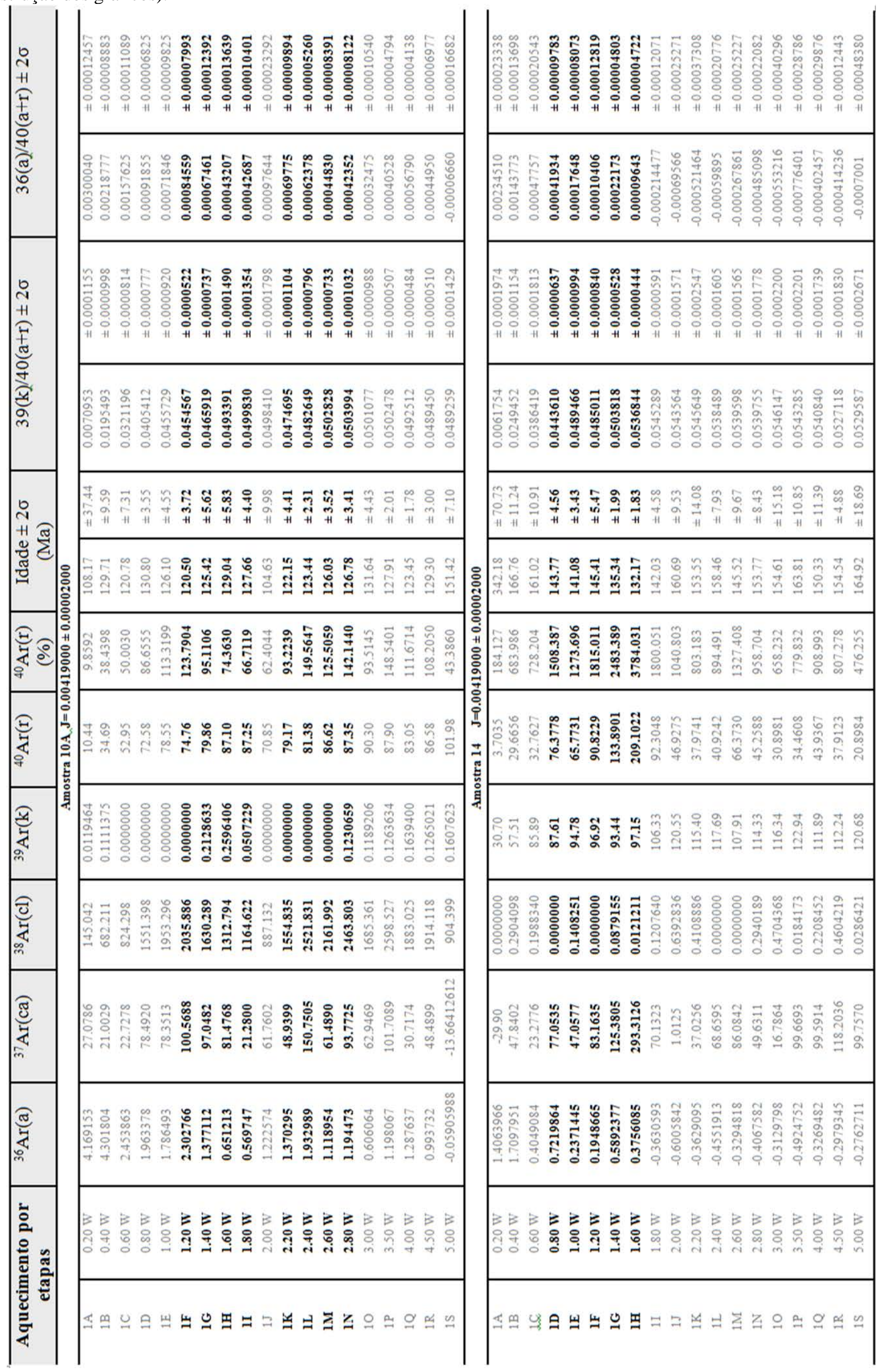


Em função de uma das etapas de aquecimento ter fornecido uma idade aparente muito jovem (etapa J) quando comparada às demais etapas de aquecimento, foram gerados dois gráficos complementares (miniplateau1, miniplateau2) cada um deles isolando o conjunto de etapas antes e depois de J. As idades plateau foram $124.69 \pm 4.23 \mathrm{Ma}$ $\left(3.39 \% 2 \sigma ; 21 \%{ }^{39} \mathrm{Ar}\right)$ e $124.50 \pm 2.24 \mathrm{Ma}$ ( $1.8 \% \quad 2 \sigma ; 30.3 \%$ 39Ar), em absoluta concordância com a idade plateau obtida considerando-se os dois conjuntos (a etapa $\mathrm{J}$ não esta incluída no cálculo da idade plateau conjunta). A idade obtida através do método de isócrona inversa foi de $131.92 \pm 3.63 \mathrm{Ma}$ (erro de $2.75 \%, 2 \sigma$ ).

A fração de rocha total da amostra 14 (diques Transminas) foi analisada também durante 19 etapas de aquecimento, com intervalo de $0.1 \mathrm{~W}$ para a potência do laser aplicada. No gráfico de aquecimento gradual
(Figura 4B) excluindo-se as três etapas iniciais (mais antigas que somam $7.1 \%$ do ${ }^{39} \mathrm{Ar}$ liberado), as etapas $\mathrm{D}-\mathrm{H}$ mostram idade combinada de $135.72 \pm 4.35$ Ma (erro de 3.37 $\%, 2 \sigma, 50.4 \%$ de ${ }^{39} \mathrm{Ar}$ liberado).

As demais etapas de mais alta temperatura (I - S) se superpõem a $2 \sigma$ e, juntas, somam $42.5 \% \quad{ }^{39} \mathrm{Ar}$, porém não sendo conceitualmente consideradas um plateau, em virtude do fato das leituras serem menores que o background, gerando valores negativos de razão ${ }^{36} \mathrm{Ar} /{ }^{40} \mathrm{Ar}$. Deste modo, as etapas de I ate $\mathrm{S}$ foram descartadas do cálculo tanto da idade plateau quanto da isócrona inversa.

A idade de isócrona inversa fornecida pelo gráfico (Figura 4B) é de $128.43 \pm 8.46 \mathrm{Ma}$ (erro de $5.90 \%, 2 \sigma$ ). Os dados das análises isotópicas das amostras $10 \mathrm{~A}$ e 14 encontram se na tabela 1 , sendo que as duas últimas colunas desta tabela se referem à isócrona inversa.

\section{DISCUSSÕES E CONCLUSÕES}

As análises de Ar-Ar são muito sensíveis às interferências das fases minerais presentes na rocha, assim como seus produtos de alteração. As perturbações nos espectros de Ar mostrados nos gráficos como o step J da amostra 10A e os dados abaixo do background da amostra 14 devem-se provavelmente à presença de fases alteradas como minerais hidratados nas amostras, como é o caso da olivina alterando-se para iddingsita (Figura 3A), bem como a sutil argilização das ripas de plagioclásio (Figura 3B).

Esta perturbação se reflete ainda na ligeira discrepância entre as idades plateau e de isócrona inversa, pois os métodos de idade plateau e de isócrona inversa possuem sensibilidades diferentes quanto às fases gasosas liberadas, sendo o método de isócrona inversa mais sensível a variações, já que nele pode se discriminar a influência da incorporação de argônio atmosférico (Kuiper, 2002).

As idades encontradas constam sumarizadas na tabela 2, e ainda que haja uma ligeira discrepância entre as idades plateau e isócrona inversa, seus valores indicam que as rochas estudadas neste trabalho realmente estão no mesmo contexto geodinâmico das demais rochas do Cretáceo Inferior que compõem a
LIP Paraná-Etendeka, cujas idades encontramse na tabela 3 . Na tabela 3 não foram apresentadas as idades juro-triássicas $\mathrm{K}$-Ar de 170-220 Ma (Dussin et al., 1995) para alguns diques de Minas Gerais, as quais podem estar associados com evento extensional anterior ao relacionado aos diques aqui datados, como por exemplo aquele marcado pelos diques Cassiporé de 176-225 Ma do Amapá (Teixeira, 1978 citado em Asmus, 1984).

As características petrográficas dos diques do enxame Transminas e Serra do Mar são as mesmas dos demais diques dos enxames eocretáceos Florianópolis, Ponta Grossa e Vitória-Colatina descritos por Coutinho (2008), e se assemelham às feições mineralógicas dos basaltos Serra Geral descritos por Bellieni et al. (1984), apesar destes basaltos terem textura afanítica diferente da fanerítica fina dos diques Transminas e Serra do Mar. Além disso, Coelho \& Chaves (2016) mostram que os diques aqui estudados possuem as mesmas características litoquímicas dos basaltos Serra Geral, tais como caráter toleítico de alto titânio e assinatura de basaltos de ilhas oceânicas (OIB-Ocean island basalts) típica de plumas mantélicas. 
Tabela 2 - Sumário das idades plateau e isócrona inversa para os diques máficos mesozoicos de Minas Gerais (P = Probabilidade estatística. MSWD = mean square weighted deviation).

\begin{tabular}{|c|c|c|c|c|c|c|c|c|}
\hline \multicolumn{6}{|c|}{ Idade Plateau } & \multicolumn{3}{|c|}{ Idade de Isócrona Inversa } \\
\hline Amostra & Material & $\mathrm{Ma} \pm 2 \sigma$ & MSWD & $\mathrm{P} \%$ & ${ }^{39} \mathrm{Ar}$ liberado \% & $\mathrm{Ma} \pm 2 \sigma$ & MSWD & $\mathrm{P} \%$ \\
\hline $10 \mathrm{~A}$ & Rocha Total & $124.56 \pm 2.15$ & 1.96 & 6 & 53.7 & $131.92 \pm 3.63$ & 0.67 & 67 \\
\hline 14 & Rocha Total & $135.72 \pm 4.35$ & 12.46 & 0 & 50.4 & $128.43 \pm 8.46$ & 3.21 & 2 \\
\hline
\end{tabular}

Tabela 3 - Sumário das idades da LIP Paraná- Etendeka e seus diques máficos associados, disponíveis na literatura.

\begin{tabular}{l|l|l}
\hline Magmatismo & Idade/Método & Referência \\
\hline Paraná-Etendeka & $135-133 \mathrm{Ma} / \mathrm{U}-\mathrm{Pb}$ & Pinto et al., 2010 \\
\hline Ponta Grossa & $134 \mathrm{Ma} / \mathrm{K}-\mathrm{Ar} / \mathrm{Ar}-\mathrm{Ar}$ & Tomba, 2012 \\
\hline Florianópolis & $134 \mathrm{Ma} / \mathrm{U}-\mathrm{Pb}$, & Florisbal et al., 2011 \\
\hline Serra do Mar & $134 \mathrm{Ma} / \mathrm{K}-\mathrm{Ar} / \mathrm{Ar}-\mathrm{Ar}$ & Tomba, 2012 \\
\hline Vitoria-Colatina & $128-136 \mathrm{Ma} / \mathrm{Ar}-\mathrm{Ar}$ & Novais et al., 2003; Belém, 2014. \\
\hline Transminas & $127 \mathrm{Ma} \mathrm{K-Ar;} \mathrm{130Ma/Ar-Ar.}$ & Pinese, 1997; Rosset et al., 2007. \\
\hline
\end{tabular}

As características petrográficas dos diques do enxame Transminas e Serra do Mar são as mesmas dos demais diques dos enxames eocretáceos Florianópolis, Ponta Grossa e Vitória-Colatina descritos por Coutinho (2008), e se assemelham às feições mineralógicas dos basaltos Serra Geral descritos por Bellieni et al. (1984), apesar destes basaltos terem textura afanítica diferente da fanerítica fina dos diques Transminas e Serra do Mar. Além disso, Coelho \& Chaves (2016) mostram que os diques aqui estudados possuem as mesmas características litoquímicas dos basaltos Serra Geral, tais como caráter toleítico de alto titânio e assinatura de basaltos de ilhas oceânicas (OIB-Ocean island basalts) típica de plumas mantélicas. A disposição espacial dos enxames de diques máficos associados à LIP ParanáEtendeka mostrada na figura $1 \mathrm{~A}$ remete a um padrão radial de instalação dos diques, o qual sugere que o mecanismo gerador desta província ígnea esteve relacionado à atividade da pluma mantélica Tristão da Cunha (Figura 1B) quando o supercontinente Gondwana derivou por sobre ela, conforme também advogam White \& McKenzie (1989), Renne et al. (1992), O’Connor et al., (1990), Harry et al. (1992), Valente et al.(2007, 2009), Corval (2009) e Chaves (2014).

Entretanto, o modelo de gênese desta LIP ainda é tema de discussão, visto que alguns autores como Marques (2001) e Ussami et al. (2012) defendem que o processo magmático se deu por delaminação do manto litosférico subcontinental, englobado por células comvectivas ascendentes do manto sublitosférico subjacente em níveis astenosféricos pouco profundos. Estes fenômenos teriam ocorrido durante um estágio avançado de rifteamento do supercontinente Gondwana.

\section{AGRADECIMENTOS}

Ao colegiado de pós-graduação do Instituto de Geociências IGC/UFMG pelo apoio financeiro e a Petrobras pela bolsa do programa Petrobras de formação de recursos humanos (PFRH). Em especial, os autores agradecem à Profa . Maria Helena Bezerra Maia de Hollanda pela execução das análises geocronológicas Ar-Ar e discussões sobre o artigo.

\section{REFERÊNCIAS}

ASMUS, H. E. Geologia da margem continental brasileira. In: SCHOBBENHAUS, C.; CAMPOS, D.A.; DERZE, G.R.; ASMUS, H.E. (cords). Geologia do Brasil. Texto explicativo do mapa geológico do Brasil e da área oceânica adjacente incluindo depósitos minerais. Brasília: DNPM. p.443-472, 1984.

BELÉM, J. Geoquímica, Geocronologia e contexto geotectônico do magmatismo máfico associado ao feixe de fraturas Colatina, Estado do Espírito Santo. Belo Horizonte, 2014. 134p. Tese (Doutorado), Instituto de Geociências, Universidade Federal de Minas Gerais.
BELLIENI, G.; COMIN-CHIARAMONTI, P.; MARQUES, L. S.; MELFI, A.J .; PICCIRILLO, E. M., NARDY, A. J. R., ROISENBERG, A. High and low-Ti flood basalts from the Paraná plateau (Brazil): petrology and geochemical aspects bearing on their mantle origin. Neues Jahrbuch für Mineralogie, v. 150, p. 272-306, 1984.

CHAVES, A. O. \& NEVES, J. M. C. Radiometric ages, aeromagnetic expression, and general geology of mafic dykes from southeastern Brazil and implications for African-South American correlations. Journal of South American Earth Sciences, v. 19, p. 387-397, 2005. 
CHAVES, A. O. Enxames de diques máficos de Minas Gerais o estado da arte. Geonomos, v. 21, p. 1, 29-33, 2013.

CHAVES, A. O. Petrografia e litoquímica do enxame de diques máficos eocretáceos Transminas (Minas Gerais, Brasil): implicações geodinâmicas. Comunicações Geológicas n. 101, Especial I, p. 163-167, 2014.

COELHO, R. M. \& CHAVES, A. O. Petrografia e litoquímica de diques máficos mesozóicos e cambrianos (?) de Minas Gerais: Comparações com basaltos da Província ParanáEtendeka e com diques cambrianos da Suite Fundão. Geonomos (Aceito para publicação), 2016.

CORVAL, A. V. Petrogênese das suítes basálticas toleíticas do Enxame de Diques da Serra do Mar nos setores central e norte do estado do Rio de Janeiro. Rio de Janeiro, 2005. 92p. Dissertação (Mestrado), Universidade Federal do Rio de Janeiro.

CORVAL, A. V. Petrogênese e contexto geodinâmico das suítes basálticas toleíticas (de alto-Tion e baixo-Tion) do Cretáceo Inferior da formação centro-oriental do enxame de diques da Serra do Mar. Rio de Janeiro, 2009. 188p. Tese (Doutorado), Universidade Federal do Rio de Janeiro.

COUTINHO, J. M. V. Dyke Swarms of the Paraná Triple Junction, Southern Brazil. Geologia USP - Série Científica, v. 8, n. 2, 29-52, 2008.

CPRM/CODEMIG. Estado de Minas Gerais-Mapa Geológico e Mapa de Recursos Minerais em Sistema de Informação Geográfica/SIG. Escala 1: 1000000. Serviço Geológico do Brasil/CPRM e Companhia de Desenvolvimento Econômico de Minas Gerais/ CODEMIG. DVD Rom, 2014

DUTRA, T. Petrogênese dos basaltos de baixo-TiO2 do Enxame de Diques da Serra do Mar na Região dos Lagos, RJ. Rio de Janeiro, 111p. 2006. Dissertação (Mestrado), Universidade Federal do Rio de Janeiro,

DUSSIN, T. M.; DUSSIN, I. A.; CHARVET, J.; BONHOMME, M. G. K-Ar Chronology of mesozoic dyke swarm from Southern Espinhaço Region (SE Brazil). Journal of South American Earth Sciences, v. 8, v. 1, p. 47-54, 1995.

FLORISBAL, L. M.; JANASI, V. M.; HEAMAN, L.M.; NARDI, L. V. S.; BITENCOURT M. F. O enxame de diques Florianópolis na região de Garopaba, SC: Idade U-Pb TIMS em Badeleita e relação com vulcanismo Serra Geral. In: SIMPÓSIO DE VULCANISMO E AMBIENTES ASSOCIADOS 5, 2011, Goiás. Resumos...Goiás: Sociedade Brasileira de Geologia, CD-ROM.

HALLS, H. C. \& FAHRIG, W. F. (Eds.). Mafic Dyke Swarms. Geological Association of Canada. Special Paper, 34p.. 1987.

HEILBRON, M.; PEDROSA-SOARES, A. C.; CAMPOS NETO, M. C.; SILVA, L. C.; TROW, R. A. J.; JANASI, V. A. Província Mantiqueira. In: MANTESSO-NETO, V.; BARTORELLI, A.; CARNEIRO, C.D.R.; BRITO-NEVES, B.B. (eds.), Geologia do Continente Sul-Americano: Evolução da obra de Fernando Flávio Marques de Almeida. Editora Beca, São Paulo, p. 203-234, 2004.

HARRY, D. L \& SAWYER, D. S. Basaltic Volcanism, mantle plumes, and the mechanics of rifting: The Paraná flood basalt province of South America. Geology, v. 20, p. 207-210, 1992.

MARQUES, L.S. Geoquímica dos diques toleíticos da costa sul-sudeste do Brasil: contribuição ao conhecimento da Província Magmática do Paraná. São Paulo, 2001. 86p. Tese (Livre-docência), Instituto de Astronomia, Geofísica e Ciências Atmosféricas, Universidade de São Paulo.

KUIPER, Y. D. The interpretation of inverse isochron diagrams in $40 \mathrm{Ar} / 39 \mathrm{Ar}$ geochronology. Earth and Planetary Science Letters, v. 203, p 499-506, 2002.

NOVAIS, L. C. C.; TEIXEIRA L. B.; RODARTE J. B. M.; ALMEIDA J; C. H.; VALERIANO C. M. Novas ocorrências de diques de diabásio na faixa Colatina- ES: estruturas rúpteis associadas e implicações tectônicas para as bacias de Campos e Espirito Santo. Boletim de Geociências da Petrobrás, v. 12, n. 1, p. 191-194, 2003.

O'CONNOR, J. M. \& DUNCAN, A. R. Evolution of the Walvis Ridge-Rio Grande Rise hot Spot System: Implication for African and South American Plate Motions Over Plumes. Journal of Geophysical Research, v. 95, n. B11, p. 17475$17502,1990$.

OLIVEIRA, E. P. \& MONTES, M. L. Os enxames de diques máficos do Brasil. SBG, Anais do $33^{\circ}$ Congresso Brasileiro Geologia, Rio de Janeiro-RJ., IX :4137-4154. 1984.

PINESE, J. P. P. Geologia, geoquímica isotópica e aspectos petrológicos dos diques máficos pré-cambrianos da região de Lavras (MG), porção sul do Cráton do São Francisco. São Paulo, 1997. 178 p. Tese (Doutorado), Instituto de Geociências, Universidade de São Paulo.

PINTO, V. M.; HARTMANN, L. A.; SANTOS, J. O. S.; MCNAUGHTON, N. J.; WILDNER, W.. Zircon U-Pb geochronology from the Paraná bimodal volcanic province support a brief eruptive cycle at $\sim 135 \mathrm{Ma}$. Chemical Geology, v. 281, p. 93-102, 2010.

RENNE, P. R.; ERNESTO, M.; PACCA, I. G.; COE, R. S.; GLEN, J. M; PRÉVOT, M.; PERRIN, M. The age of Paraná flood volcanism, rifting of Godwanaland, and the JurassicCretaceous Boundary. Science, v. 258, p. 975-978, 1992.

RENNE, P. R.; MUNDIL, R.; BALCO, G.; MIN, K.; LUDWIG, K. R. 2010. Joint determination of 40K decay constants and $40 \mathrm{Ar} * / 40 \mathrm{~K}$ for the Fish Canyon sanidine standard and improved accuracy for $40 \mathrm{Ar} / 39 \mathrm{Ar}$ geochronology. Geochemistry and Cosmochemistry Acta, 74: 5349-5367)

ROCHA, L. G. M., Caracterização magnética da porção central do lineamento azimute $\mathbf{1 2 5}^{\circ}$. Brasília, 2013. 166p. Tese (Doutorado), Universidade de Brasília.

ROSSET, A.; DE MIN, A.; MARQUES, L. S.; MACAMBIRA, M. J. B.; ERNESTO, M.; RENNE, P. R.; PICCIRILLO, E. M. 2007. Genesis and geodynamic significance of Mesoproterozoic and Early Cretaceous tholeiitic dyke swarms from the São Francisco Craton (Brazil). Journal of South American Earth Sciences, 24, 69-92.

SCHMITT, R. S.; TROUW, R. A. J.; VAN SCHMUS, W. R.; PIMENTEL, M. M. Late amalgamation in the central part of West Gondwana: new geochronological data and the characterization of a Cambrian collisional orogeny in the Ribeira Belt (SE Brazil). Precambrian Research, v. 133, p. 29-61, 2004.

SILVA, A. M.; CHEMALE, F.; KUYUMJIAN, R. M.; HEAMAN, L. Mafic dike swarms of Quadrilátero Ferrífero and southern Espinhaço, Minas Gerais, Brazil. Revista Brasileira de Geociências, v. 25, n. 2, p. 124-137, 1995.

TOMBA, C. L. B. Análise estrutural dos enxames de diques máficos eocretáceos do Sul-Sudeste do Brasil. São Paulo, 2012. 133p. Dissertação (Mestrado), Instituto de Geociências, Universidade de São Paulo.

USSAMI, N.; CHAVES, C. A. M.; MARQUES, L. S.; ERNESTO, M. Origin of the Rio Grande Rise-Walvis Ridge reviewed integrating palaeogeographic reconstruction, isotope geochemistry and flexural modelling. Geological Society, London, Special Publications, v. 369, n 1, 129-146, 2012.

VALENTE, S. C. Geochemical and isotopic constraints on the petrogenesis of the Cretaceous dykes of Rio de Janeiro, Brazil. Belfast, 1997. 366p. Tese (Doutorado). The Queen's University of Belfast.

VALENTE, S. C.; CORVAL, A.; DUARTE, B. P.; ELLAM, R. B.; FALLICK, A. E.; MEIGHAN, I. G.; DUTRA, T. Tectonic boundaries, crustal weakness zones and plume-subcontinental lithospheric mantle interactions in the Serra do Mar Dyke Swarm, SE Brazil. Revista Brasileira de Geociências, v. 37, 
p. 194-201, 2007.

VALENTE, S. C.; DUTRA, T.; HEILBRON, M.; CORVAL, A.; SZATMARI, P. Litogeoquímica de diques de diabásio da faixa Colatina, ES. Geochimica Brasiliensis, v. 23, n. 2, p 177-192, 2009

WHITE, R. S. \& MCKENZIE, D. Magmatism at rift zones: the generation of volcanic continental margins and flood basalts.
Journal of Geophysical Research, v. 94, p. 7685-7729, 1989.

Submetido em 22 de janeiro de 2016 Aceito em 31 de julho de 2017 